\title{
Seeking clarity: Insights from a highly effective preparation protocol for suppressing myocardial glucose uptake for PET imaging of cardiac inflammation
}

\author{
Michael T. Osborne, $M D,{ }^{a}$ and Sanjay Divakaran, $M D^{b}$ \\ a Department of Radiology and Cardiology Division, Cardiovascular Imaging Research Center, \\ Massachusetts General Hospital and Harvard Medical School, Boston, MA \\ b Cardiovascular Imaging Program, Departments of Radiology and Medicine and Division of \\ Cardiovascular Medicine, Department of Medicine, Brigham and Women's Hospital and \\ Harvard Medical School, Boston, MA
}

Received Aug 12, 2019; accepted Aug 12, 2019

doi: $10.1007 / \mathrm{s} 12350-019-01864-z$

\section{See related article, pp. 849-861}

As ${ }^{18}$ F-fluorodeoxyglucose positron emission tomography $\left({ }^{18} \mathrm{~F}\right.$-FDG PET) is increasingly used for the detection of pathologic myocardial inflammation, a growing body of research has focused on improving imaging quality by seeking strategies to more successfully suppress background myocardial uptake of ${ }^{18} \mathrm{~F}$ FDG, a radioactive glucose analogue. Both inflammatory cells and normal myocytes are highly metabolically active. However, unlike inflammatory cells, which constitutively rely on glucose for metabolism, normal myocytes are able to use either glucose or free fatty acids to meet their metabolic demands, depending on their current metabolic state. ${ }^{1,2}$ As such, patients must undergo preparation to shift background myocardial metabolism toward free fatty acids prior to radiotracer injection in order to effectively visualize pathologic inflammatory ${ }^{18}$ F-FDG uptake within the heart. To achieve this, a recent joint Society of Nuclear Medicine and Molecular Imaging-American Society of Nuclear Cardiology (SNMMI-ASNC) consensus statement provides broad rather than specific recommendations for

Reprint requests: Michael T. Osborne, MD, Department of Radiology and Cardiology Division, Cardiovascular Imaging Research Center, Massachusetts General Hospital and Harvard Medical School, 55 Fruit St., Yawkey 5E, Boston, MA 02114-2750; mosborne@partners.org J Nucl Cardiol 2020;27:862-4.

$1071-3581 / \$ 34.00$

Copyright (c) 2019 American Society of Nuclear Cardiology. patient preparation as the existing literature describes markedly heterogeneous protocols with no consensus on what defines adequate suppression. ${ }^{3,4}$ While there are clearly multiple ways to minimize myocyte glucose utilization, further insights are needed to develop an evidence-based, optimized protocol that can be broadly implemented across sites to maximize the utility of this imaging technique.

The current article by Larson et al. provides important additional details and suggestions as we progress toward this goal. ${ }^{5}$ The implemented protocol incorporates several strategies to minimize myocardial ${ }^{18}$ F-FDG uptake, including the use of a low carbohydrate/high fat diet, a prolonged fast, a high fat drink on the day of imaging, and boluses of intravenous heparin. There was a very high rate of patient adherence to this stringent protocol which resulted in a $95 \%$ rate of adequate suppression and diagnostic ${ }^{18}$ F-FDG PET imaging among the included 111 patients from the University of Michigan with suspected cardiac inflammation. This rate of efficacy is similar to that reported in a recent study from the Mayo Clinic that implemented a preparation strategy based on the recent SNMMI-ASNC consensus statement on ${ }^{18}$ F-FDG PET myocardial imaging. ${ }^{3,6,7}$ Additionally, the current study provides serologic evaluation of key metabolic parameters including glucose, free fatty acid, C-peptide, and insulin levels. Through these measurements, the authors demonstrated significant associations between standardized uptake values (SUVs) of the blood pool and myocardium with markers of glucose metabolism as well as a lack of an association between the same SUVs and fatty acid metabolism. The authors argue, therefore, that modification of glucose 
metabolism may have a more important role in myocardial ${ }^{18}$ F-FDG uptake suppression with the described protocol.

A key success of this manuscript is establishing normative values for metabolic parameters using this protocol. Because of the intricacies of this study and variability between this protocol and those previously described, it is quite possible that the reported values may apply specifically to this particular protocol. Nevertheless, these values may provide an objective serological measurement that associates with the adequacy of preparation with this protocol prior to radiotracer injection. Importantly, a recent study examining the reproducibility of myocardial ${ }^{18}$ F-FDG PET imaging using a different preparation protocol showed that while measurements of glucose and fatty acid metabolism on serial studies were similar, there was not a consistent relationship between these values and imaging findings. ${ }^{8}$ As such, further evaluation of metabolic parameters resulting from other preparation strategies and the relationship between these measurements and imaging findings is needed to determine whether the values specified herein are both meaningful and broadly applicable.

Another important component of the implemented protocol was its clear delineation to patients and the reinforcement of adherence via questionnaires and physician review. Stringent review of adherence to the preparation protocol prior to ${ }^{18} \mathrm{~F}$-FDG injection is critical and has been employed with success in multiple recent studies describing highly effective preparation protocols. $^{5,6}$ This strategy does not require substantial effort and can only help to minimize unnecessary testing and radiotracer administration for patients. Appropriately, such a review is emerging as an integral component of any preparation protocol.
Another success of the current study is the identification of the ratio of myocardial to blood pool SUV as a potential imaging marker of adequate suppression. At present, the determination of adequate suppression is largely subjective; however, Larson et al. identified the ratio of myocardial to blood pool SUV as a potentially useful and much needed objective means of describing the adequacy of preparation. This measurement may be universally relevant, and its assessment can be easily integrated into reports to standardize the assessment of background myocardial ${ }^{18}$ F-FDG uptake. As with serological measurements of metabolism, the utility of this marker and the normative values using other preparation protocols require additional study.

While several key observations are provided by this manuscript, it should be noted that the described protocol is somewhat labor intensive, and there is no comparison available to determine the effectiveness of the described protocol versus one that coincides more closely with that proposed by the recent SNMMI-ASNC consensus statement on ${ }^{18} \mathrm{~F}-\mathrm{FDG}$ PET myocardial imaging. ${ }^{3}$ Notably, 41 patients $(27 \%$ of the overall cohort) did not complete the protocol as described and were not included in this analysis. As such, it is possible that the included population may not reflect the protocol's true effectiveness since these individuals with protocol deviations, which are likely to occur with broader implementation of a complex protocol, were not included. Even still, it is important that the efficacy of the described protocol was similar among those included who are part of populations with unique challenges for imaging preparation (e.g., those imaged as inpatient or those with diabetes or heart failure). ${ }^{9}$ Furthermore, prior research has resulted in uncertainty with regard to the benefit of using a high fat drink and/or intravenous heparin as part of a preparation strategy for background

Table 1. Opportunities to optimize positron emission tomography imaging of cardiac inflammation

\begin{tabular}{ll}
${ }^{18}$ F-Fluorodeoxyglucose Ideal tracer \\
\hline
\end{tabular}

Preparation protocols to decrease prevalence of focal on diffuse pattern

Identification of an objective measurement of myocardial suppression (imaging and/or serologic)

Implementation of pre-imaging review of patient preparation to determine adherence

Evaluation of study reproducibility and repeatability of preparation protocols

Multi-center trial comparing preparation protocols to identify the most simple and effective strategy
More specific for inflammatory cells

No requirement for suppression of normal myocardial tracer uptake

Simplified preparation protocol for patients and staff 
myocardial suppression. ${ }^{4}$ Since a high fat drink and heparin would theoretically serve to primarily increase the concentration of free fatty acids rather than modify glucose metabolism, the results of the current study do not entirely reinforce the use of these strategies given the findings discussed above that suggest greater importance for the manipulation of myocardial glucose metabolism.

Two of the greatest challenges that remain in ${ }^{18} \mathrm{~F}$ FDG PET myocardial imaging are how to prevent focal on diffuse myocardial ${ }^{18} \mathrm{~F}$-FDG uptake and how to interpret this pattern. The authors should be commended on providing thumbnail images from all 111 studies in the Supplemental Material for general review. Impressively, only four of the $111(3.6 \%)$ studies had a focal on diffuse uptake pattern. ${ }^{5}$ However, half of those were interpreted as diagnostic, and the other half were interpreted as non-diagnostic. This highlights how difficult it is to provide helpful information to referring colleagues and patients when this pattern is seen. In this situation, a diagnostic determination often hinges on the clinical history and presence of extracardiac findings consistent with sarcoidosis. As the quest for tracers that are more specific for inflammatory cells ${ }^{10}$ and/or do not require myocardial substrate manipulation goes on, rarifying the focal on diffuse pattern will be a critical advance in ${ }^{18} \mathrm{~F}$ FDG PET imaging of known or suspected cardiac sarcoidosis.

In summary, Larson et al. have provided us with another careful and effective patient preparation method to suppress myocardial glucose utilization in ${ }^{18} \mathrm{~F}$-FDG PET myocardial imaging and have provided potentially important and novel insights that clarify the pattern of metabolic and imaging parameters that indicate adequate suppression. Nevertheless, further opportunities to improve remain (Table 1). Given our continued reliance on ${ }^{18}$ F-FDG PET for diagnosing and treating inflammatory cardiac conditions and our need for further clarity, perhaps it is finally time to study and compare different patient preparation protocols in a randomized, multi-center trial. While we have been highly successful at identifying a variety of strategies that effectively minimize background myocardial uptake, we now should come together as a community and systematically determine which of these strategies is best.

\section{Disclosures}

Neither author has any significant disclosures related to this work.

\section{Funding}

This work is supported in part by the following Grants: United Stated National Institutes of Health (NIH) \#KL2TR002542 to Dr. Osborne and \#T32HL094301 to Dr. Divakaran.

\section{References}

1. Depre C, Vanoverschelde JL, Taegtmeyer H. Glucose for the heart. Circulation 1999;99:578-88.

2. Mochizuki T, Tsukamoto E, Kuge Y, Kanegae K, Zhao S, Hikosaka K, et al. FDG uptake and glucose transporter subtype expressions in experimental tumor and inflammation models. J Nucl Med 2001;42:1551-5.

3. Chareonthaitawee P, Beanlands RS, Chen W, Dorbala S, Miller EJ, Murthy VL, et al. Joint SNMMI-ASNC expert consensus document on the role of (18)F-FDG PET/CT in cardiac sarcoid detection and therapy monitoring. J Nucl Cardiol 2017;24:174158.

4. Osborne MT, Hulten EA, Murthy VL, Skali H, Taqueti VR, Dorbala S, et al. Patient preparation for cardiac fluorine-18 fluorodeoxyglucose positron emission tomography imaging of inflammation. J Nucl Cardiol 2017;24:86-99.

5. Larson SR, Pieper JA, Hulten EA, Ficaro EP, Corbett JR, Murthy VL, et al. Characterization of a highly effective preparation for suppression of myocardial glucose utilization. J Nucl Cardiol 2019;8:005996. https://doi.org/10.1161/JAHA.117.005996.

6. Christopoulos G, Jouni H, Acharya GA, Blauwet LA, Kapa S, Bois J, et al. Suppressing physiologic 18-fluorodeoxyglucose uptake in patients undergoing positron emission tomography for cardiac sarcoidosis: The effect of a structured patient preparation protocol. J Nucl Cardiol 2019. https://doi.org/10.1007/s12350-01 9-01746-4.

7. Divakaran S, Osborne MT. Preparation is everything: The impact of a structured preparation protocol on cardiac (18)F-FDG PET imaging for cardiac sarcoidosis. J Nucl Cardiol 2019. https://doi. org/10.1007/s12350-019-01788-8.

8. Alvi RM, Young BD, Shahab Z, Pan H, Winkler J, Herzog E, et al. Repeatability and optimization of FDG positron emission tomography for evaluation of cardiac sarcoidosis. JACC Cardiovasc Imaging 2019;12:1284-7.

9. Davila-Roman VG, Vedala G, Herrero P, de las Fuentes L, Rogers JG, Kelly DP, et al. Altered myocardial fatty acid and glucose metabolism in idiopathic dilated cardiomyopathy. J Am Coll Cardiol 2002;40:271-7.

10. Divakaran S, Stewart GC, Lakdawala NK, Padera RF, Zhou W, Desai AS, et al. Diagnostic accuracy of advanced imaging in cardiac sarcoidosis. Circ Cardiovasc Imaging 2019;12:e008975.

Publisher's Note Springer Nature remains neutral with regard to jurisdictional claims in published maps and institutional affiliations. 